\title{
Horse rug lung: toxic pneumonitis due to fluorocarbon inhalation
}

G M F Wallace, P H Brown

Occup Environ Med 2005;62:414-416. doi: 10.1136/oem.2004.015784

Fluorocarbons are widely used in industry, and manifestations of inhalation toxicity include polymer fume fever, reactive airways dysfunction, and bronchospasm. Only seven cases of alveolitis occurring acutely after inhalation have been reported. This paper presents four cases of toxic pneumonitis due to direct inhalation of industrial fluorocarbon used as a waterproofing spray for horse rugs. These cases differ from previous reports and show that chronic as well as acute alveolitis can result from fluorocarbon inhalation. Corticosteroid treatment may be beneficial. The need for stricter control in the workplace is emphasised.

$\mathrm{F}$ luorocarbons are used in industry for a wide range of applications. These include use as mechanical and electrical components, in fibreoptics, as a non-stick agent in the manufacturing of cooking utensils, and as oil, water, and stain repellents in upholstery production. Inhalation toxicity manifests in a variety of ways. Polymer fume fever is the best described. It presents within hours of exposure with dyspnoea, pyrexia, nausea and vomiting, unproductive cough, peripheral blood leucocytosis, hypoxaemia, and alveolar infiltrates on chest $x$ ray and CT scan. ${ }^{1-5}$ Transbronchial biopsy shows alveolitis ${ }^{1}$ and alveolar collapse. ${ }^{5}$ Complete resolution of symptoms occurs within days. ${ }^{1-5}$ Reactive airways dysfunction and acute respiratory distress syndrome have also been reported. ${ }^{67}$ There have been only seven reported cases of alveolitis, all occurring acutely after inhalation of leather impregnation spray in a confined space. $^{89}$ All had pulmonary oedema on chest $x$ ray and disorders of ventilation and diffusion. Six patients received intravenous steroids and showed complete resolution of the alveolitis. The remaining patient did not receive steroids and developed pulmonary fibrosis.

This paper reports three directly linked cases and a probable fourth case of toxic pneumonitis due to inhalation of a fluorocarbon based waterproofing agent, whose presentations are unlike those previously described.

\section{CASE REPORTS}

All four patients worked for a horse rug cleaning firm which employed 14 people. Following laundering, a fluorocarbon polymer spray (Rucoguard EPF 1619) was applied as a waterproofing agent. This is a cationic fluorocarbon polymer made by telomerisation, the precise contents of which are a trade secret. Only one of the contents, isopropranol, has an occupational exposure standard. Spraying was undertaken in an $8 \times 8 \times 10$ foot booth using a spray gun connected to an air compressor. The booth had a single extractor fan in the ceiling and the walls and floor were lined with residue of the fluorocarbon polymer plus horsehair. A filter mask, precise specification unclear, was available. The door was frequently left open, allowing fumes to escape into the adjacent factory environment where rugs were washed and packaged.

A 23 year old man, a light smoker with no previous medical history, dismantled the air compressor inside the booth without respiratory protection during his first week of work. Compressed air escaped and disturbed the fluorocarbon residue on walls and floor, producing a fume and dust cloud. He left the booth but within 30 minutes developed breathlessness and "throat tightness". At hospital he had frequent cough, tachypnoea ( 33 breaths per minute), was cyanosed, and unable to speak sentences. There was no fever, angioedema, or stridor, he had no wheezes or lung crackles, and cardiac examination was normal. Arterial blood gases (ABG) showed type 1 respiratory failure $\left(\mathrm{pH} 7.42, \mathrm{pO}_{2}\right.$ $7.5 \mathrm{kPa}, \mathrm{pCO}_{2} 4.7 \mathrm{kPa}$, oxygen saturation $89.5 \%$ ), and chest $x$ ray $(\mathrm{CXR})$ examination showed bilateral alveolar shadowing. Electrocardiography was normal. High flow oxygen and intravenous hydrocortisone and chlorpheniramine were initially given. Subsequently he received $200 \mathrm{mg}$ intravenous hydrocortisone twice daily for 24 hours for suspected pneumonitis, and $50 \mathrm{mg}$ intravenous frusemide in case of pulmonary oedema, before changing to $50 \mathrm{mg}$ once daily oral prednisolone. After 24 hours his hypoxia and chest $x$ ray abnormalities had nearly resolved. He developed a peripheral blood neutrophilia but remained apyrexial. His unproductive cough and breathlessness persisted at 48 hours and pulmonary function tests showed a restrictive defect with reduced gas transfer $\left(\mathrm{FEV}_{1} 2.26 \mathrm{l}\right.$ (53\% predicted), FVC $3.38 \mathrm{l}$ (66\%), $\mathrm{FEV}_{\mathrm{l}} / \mathrm{FVC} 67 \%$, TLC $4.82 \mathrm{l}$ (70\%), RV $1.30 \mathrm{l}$ (81\%), DLCO $23.6 \mathrm{ml} / \mathrm{min} / \mathrm{mm} \mathrm{Hg}(67 \%)$. He was discharged after three days with a one week course of $50 \mathrm{mg}$ prednisolone daily.

Three weeks later he had returned to work but complained of exertional dyspnoea after half a mile, and an unproductive cough when exposed to car fumes. CXR was normal and spirometry improved $\left(\mathrm{FEV}_{1} 3.34 \mathrm{l}\right.$, FVC $\left.3.9 \mathrm{l}\right)$. An inhaled corticosteroid (budesonide $800 \mu \mathrm{g}$ daily via turbohaler) was prescribed for suspected alveolitis. His dyspnoea and cough, however, worsened and he was readmitted to hospital the following month. He was tachypnoeic ( 24 breaths/minute) at rest but examination was otherwise normal. ABGs on air, FBC, U\&Es, C reactive protein, and CXR were all normal. Pulmonary function tests showed a persistent restrictive defect $\left(F^{2} V_{1} 3.04\right.$ l, FVC 3.36 l, TLC 4.76 l, RV 1.32 l), with impaired gas transfer (DLCO $19.8 \mathrm{ml} / \mathrm{min} / \mathrm{mm} \mathrm{Hg}$ ) and no reversibility to $5 \mathrm{mg}$ nebulised salbutamol. High resolution CT (HRCT) scan of the lungs showed bilateral ground glass shadowing, mainly in the upper lobes, with no evidence of fibrosis (fig 1). Autoimmune screening and angiotensin converting enzyme (ACE) levels were normal. Fibreoptic bronchoscopy was normal and no pathogens were isolated

Abbreviations: $\mathrm{CT}$, computed tomography; DLCO, diffusing capacity for carbon monoxide; $\mathrm{FEV}_{1}$, forced expiratory volume in one second; FVC, forced vital capacity; Ig, immunoglobulin; RV, residual volume; TLC, total lung capacity 


\section{Main messages}

- Acute and chronic alveolitis can result from inhalation of industrial fluorocarbons, at least in the setting where there is co-exposure to animal hair.

- Corticosteroid therapy modifies the disease process but does not cause complete resolution.

from bronchial washings. Transbronchial biopsies showed mildly inflamed pulmonary parenchyma, interstitial thickening, foci of organising exudate, and striking proliferation of type 2 alveolar lining cells in keeping with toxic alveolar injury (fig 2). He was discharged taking $30 \mathrm{mg}$ once daily prednisolone for four weeks but did not complete the course and did not return to work. One month later spirometry showed marked improvement (FEV 4.0 l, FVC 5.4 l; DLCO not measured). He failed to attend further appointments.

During the next 15 months three other employees of the same company were referred to the chest clinic with cough and dyspnoea. Two men, aged 18 and 37 years, regularly undertook fluorocarbon spraying. A 35 year old female packaged the processed rugs nearby. The 18 year old, a smoker, had had mild symptoms for a year, which first occurred four months after starting work. These worsened over several months, although improved during holidays, and he left the job soon after the accident to the index case above. When he attended the clinic four months later his symptoms had greatly improved. He had no lung crackles, CXR was normal, but lung function tests showed a mild restrictive defect and moderate DLCO reduction (45\% predicted). He did not attend for lung HRCT or outpatient review then but did so one year later. At this time he had no symptoms, lung volumes were virtually normal, but DLCO was $54 \%$ predicted. HRCT showed focal ground glass densities with a centrilobular distribution. Transbronchial biopsies showed patchy fibrosis. Two years after stopping fluorocarbon exposure his gas transfer had not improved further.

The 37 year old, a non-smoker, had a history of allergic rhinitis and succeeded the above patients as rug sprayer. He worked for up to 11 hours daily six days per week with no holidays, using a filter helmet with separate air supply as respiratory protection. He presented with weight loss of $12 \mathrm{~kg}$ in addition to cough and dyspnoea, which had started four months after commencing work. He had no lung crackles or evidence of digital clubbing. Peripheral blood showed raised

\section{Policy implications}

- Where fluorocarbons are used in the workplace, stricter controls and a greater awareness of potential hazards are required.

titres of specific IgE to horse allergen (the only one of the four cases who had this). Lung HRCT showed predominantly upper lobe ground glass shadowing. Transbronchial biopsy specimens were of identical appearance to those described in the index case. He stopped work and his symptoms and lung function improved substantially with prednisolone $30 \mathrm{mg}$ daily, tapering over several months. Repeat HRCT was normal four months later. One year after presentation he still had impaired lung function (TLC 78\%, DLCO 66\%).

The 35 year old female, a smoker, had worked for six months when she developed cough and dyspnoea which did not respond to inhaled corticosteroids. She stopped work and at presentation to the clinic two months later, her symptoms had improved. Her CXR and HRCT were normal but she had a restrictive defect $\left(\mathrm{FEV}_{1}\right.$ 67\%; FVC 68\%; TLC 66\%;RV 66\%; DLCO $45 \%)$. Three months later her lung volumes were nearly normal but gas transfer remained low (52\%). She did not attend for follow up.

The employer was advised to inform RIDDOR (Reporting of Injuries, Diseases, and Dangerous Occurrences Regulations 1995), through which the Health and Safety Executive would be informed. In the meantime, however, the company moved to a new site, improved the degree of respiratory protection provided for spraying, and subsequently changed the method of fluorocarbon application whereby the agent was added directly to the washing machine and, after washing, the rugs were hung out to dry. To our knowledge there have been no further cases of pneumonitis among the employees.

\section{DISCUSSION}

The first case shows a clear association between heavy occupational exposure to fluorocarbon and horsehair residue and severe acute pneumonitis. The pattern of illness in the other cases is consistent with a chronic pneumonitis/ alveolitis with onset of symptoms three to six months from initial exposure and gradual resolution of a restrictive ventilatory defect after cessation of work. HRCT scans showed evidence of alveolitis in three cases. The fourth, who had the lowest exposure, being exposed to fluorocarbon
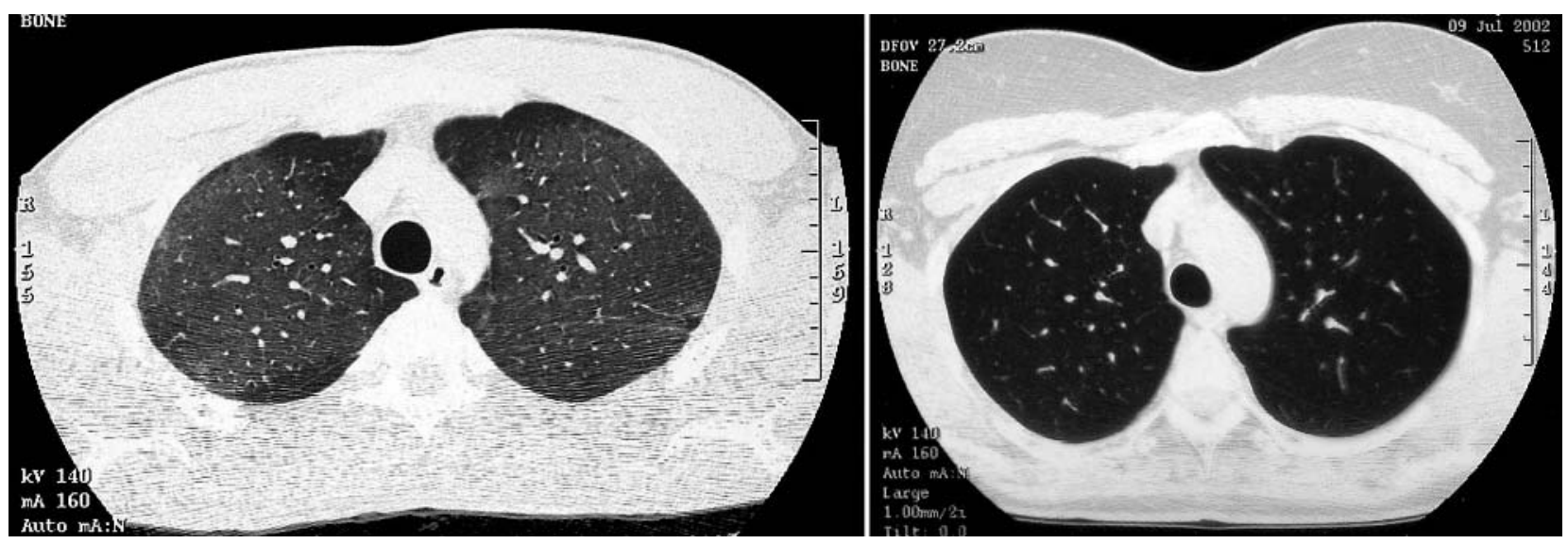

Figure 1 High resolution CT scan (HRCT) of the chest from the first case showing bilateral ground glass opacification (left) alongside a normal HRCT of the chest for comparison (right). 

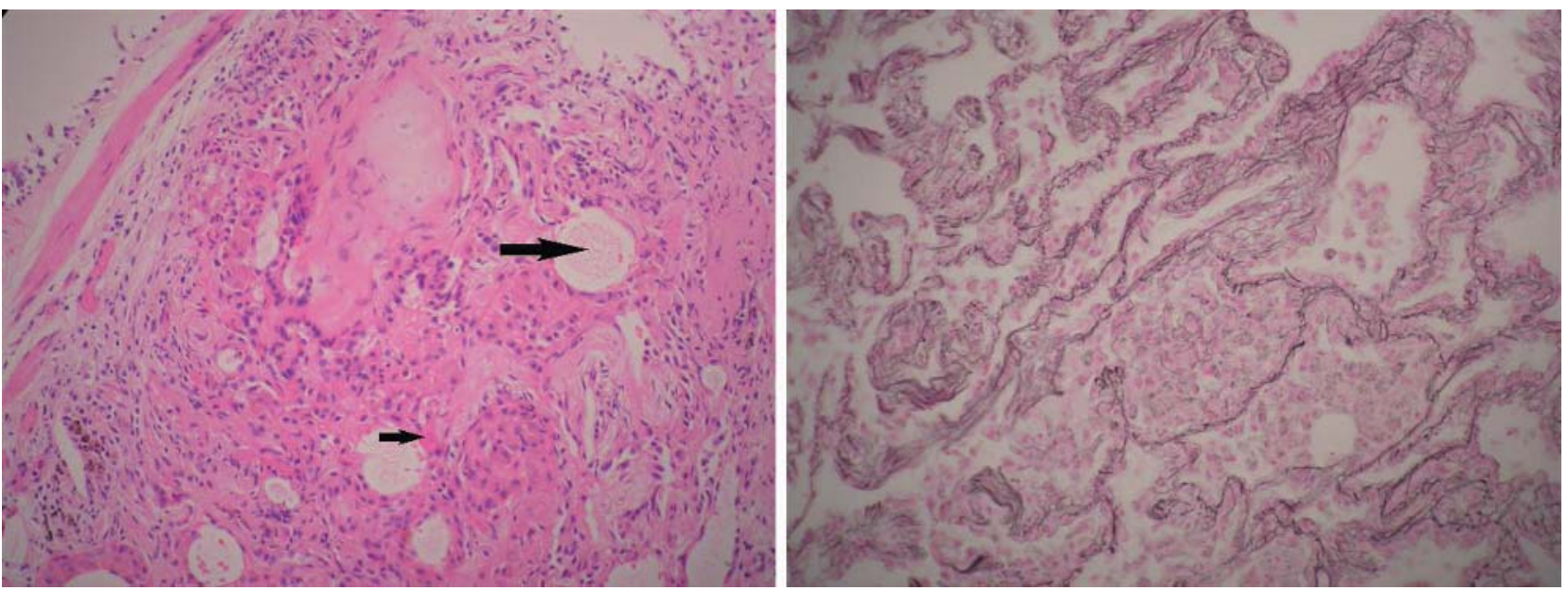

Figure 2 Haematoxylin and eosin stain (left) of lung parenchyma from the first case showing mildly inflamed pulmonary parenchyma, interstitial thickening, foci of organising exudate (large arrow), and striking proliferation of type 2 alveolar lining cells (small arrow) in keeping with toxic alveolar injury. The reticulin stain (right) from the same case confirms that there is no fibrosis. We acknowledge Dr Jenny Dhundee for her help with this.

fumes when the spray booth door was open, had a normal scan two months after stopping work, making her diagnosis less certain than that of the others. None had digital clubbing or lung crackles, nor had any evidence of obstructive airways disease. The transbronchial biopsies performed in the index case and in the 37 year old man further support the diagnosis of toxic pneumonitis. The two cases with the highest acute and chronic exposure required systemic corticosteroid therapy which appeared effective in controlling symptoms and improving lung function. Notably, the latter developed chronic pneumonitis despite augmented respiratory protection. The 18 year old, who did not receive corticosteroids, developed pulmonary fibrosis.

Exposure to horsehair may cause asthma and allergic rhinitis but is not known to cause alveolitis. ${ }^{10}$ One of the two men with chronic pneumonitis was atopic and had specific horse allergen antibodies. The other had had regular contact with horses since childhood, as had the woman. None had evidence of asthma. It seems very unlikely therefore that the alveolitis was due to horsehair exposure alone. These may be the first described cases of pneumonitis due to a toxic interaction between horsehair and fluorocarbon. Any endotoxin in the horsehair would not be denatured by the washing process. Alternatively, toxicity may be solely due to fluorocarbon exposure. Three cases smoked, but there is no known toxic interaction between cigarette smoking and fluorocarbon aerosol inhalation. Smoking may have partially contributed to reduced gas transfer in these three but HRCT scans showed no emphysema. The exact contents of the spray containing the fluorocarbon polymer are unknown so coexposures cannot be entirely excluded. The isopropranol in the polymer is not known to cause pneumonitis or to potentiate the toxicity of fluorocarbon.

We consider it very likely that the respiratory disease in these patients is due to inhalation of fluorocarbon alone or in conjunction with horsehair. There is evidence of persistently impaired lung function despite cessation of exposure. Corticosteroids may modify the disease process but it is notable that in the first case there was incomplete resolution and relapse on re-exposure. This has not been previously reported, nor has the chronic pneumonitis seen in two patients. The incomplete resolution of respiratory disease and prolonged exposure, along with lack of pyrexia, also make these cases unlike those of polymer fume fever.
Acute and chronic pneumonitis are potential hazards of spraying fluorocarbons in the workplace, at least in the setting where there is co-exposure to animal hair. It may be therefore appropriate for the Health and Safety Executive to undertake risk assessments where such co-exposure exists, and for the respiratory health of workers to be monitored. Reducing lung exposure to inhaled fluorocarbons by changing the formulation or method of application may reduce the risk of lung toxicity. Above all, a greater awareness of the potential hazards of fluorocarbons is necessary.

\section{Authors' affiliations \\ G M F Wallace, Department of Respiratory Medicine, Ninewells Hospital, Dundee, Scotland, UK \\ P H Brown, Department of Medicine, Perth Royal Infirmary, Perth, Scotland, UK \\ Competing interests: none}

Correspondence to: $\operatorname{Dr}$ G M F Wallace, Department of Respiratory Medicine, Ninewells Hospital, Dundee, Scotland, UK; gillianmfwallace@ blueyonder.co.uk

Accepted 15 January 2005

\section{REFERENCES}

1 Tanino M, Kamishima K, Miyamoto $\mathrm{H}$, et al. Acute respiratory failure caused by inhalation of waterproofing spray fumes. Nihon Kokyuki Gakkai Zasshi 1999;37:983-6.

2 Wegman DH, Peters JM. Polymer fume fever and cigarette smoking. Ann Intern Med 1974;81:55-7.

3 Silver MJ, Young DK. Acute noncardiogenic pulmonary edema due to polymer fume fever. Cleve Clin J Med 1993;60:479-82.

4 Testud F, Gabrielle L, Paquin ML, et al. Acute alveolitis after using a waterproofing aerosol: apropos of 2 cases. Rev Med Interne 1998;19:262-4.

5 Ota H, Koge K, Tanaka H, et al. Acute respiratory failure due to inhalation of aerosol water proof agent. Nihon Kokyuki Gakkai Zasshi 2000;38:485-9.

6 de la Hoz RE. Reactive airways dysfunction syndrome following exposure to a fluorocarbon. Eur Respir J 1999;13:1192-4.

7 Bracco D, Favre JB. Pulmonary injury after ski wax inhalation exposure. Ann Emerg Med 1998;32:616-19.

8 Muller-Esch G, Brunk E, Dionlagic $\mathrm{H}$, et al. Pulmonary effect of inhaling leather-impregnation sprays. Dtsch Med Wochenschr 1982;107:692-5.

9 Schicht R, Hartien A, Sill V. Alveolitis after inhalation of leather-impregnation spray. Dtsch Med Wochenschr 1982;107:688-91.

10 Tutluoglu B, Atis S, Anakkaya AN, et al. Sensitization to horsehair, symptoms and lung function in grooms. Clin Exp Allergy 2002;32:1170-3. 